\title{
PlanningLines: Novel Glyphs for Representing Temporal Uncertainties and their Evaluation
}

\author{
Wolfgang Aigner, Silvia Miksch, Bettina Thurnher, Stefan Biffl \\ Institute of Software Technology \& Interactive Systems, Vienna University of Technology \\ Favoritenstraße 9-11/188, A-1040 Vienna, Austria, Europe \\ \{aigner, silvia, thurnher, biffl\}@ifs.tuwien.ac.at
}

\begin{abstract}
Dealing with temporal uncertainties is a key issue in domains like project management or medical treatment planning. However, support for temporal indeterminacies is not very well integrated in current methods, techniques, and tools. In this paper we present a visualization technique called PlanningLines that allows for representing temporal uncertainties and aims at supporting project managers in their difficult planning and controlling tasks. We conducted a controlled experiment to gather empirical evidence on the strengths and limitations of our approach. Main results are that PlanningLine users make fewer mistakes and are faster in conducting tasks than users of a traditional visualization technique.
\end{abstract}

Keywords --- glyph, temporal data, uncertainty, usability study, project management.

\section{Introduction}

Planning future activities is a task that we have to face constantly. Since the future is always inherently connected with possible uncertainties, delays, and the unforeseen we have learned to deal with this circumstances in everyday life. Application areas such as project management or medical treatment planning have to cope with inexact knowledge about future activities and temporal uncertainties. When future planning is done in a more formal way, support for indeterminacies is not very well integrated. Furthermore, only very few visualization techniques provide means to represent such uncertainties.

Particularly, project managemers face the extremely difficult tasks of planning future activities, dealing with uncertainties, delays, estimations, meeting deadlines, managing people, assigning resources, budget constraints, and many more. In order to support project managers, many methods, tools, and techniques like PERT (Program Evaluation and Review Technique), CPM (Critical Path Method), Gantt charts, or project management software have been developed and applied. But despite the long tradition of the field, managers still have to face lots of problems - delays arise, deadlines cannot be met, expenses explode, or projects get cancelled. White \& Fortune conducted an empirical study [12] to investigate current practices in project management. They list relevant problems and critical factors, whereas the number two entry on the list of critical factors is realistic schedules. Another top listed entry on the list of limitations of current methodologies is difficulty to model 'real world'. These results indicate the need for support in dealing with temporal uncertainties in order to improve project management.

In our work, we especially focus on the temporal domain. Key tasks of project management are the estimation of task durations as well as begin and end times. On the one hand, this needs a lot of experience and many unknown factors might influence estimations. On the other hand, often different levels of uncertainty are known but cannot be integrated or dealt with using current methods. Gantt and PERT charts are the most widely used visual techniques in project management. Gantt charts, on the one hand, do not have a notion to represent indeterminacies at all. Because of this, the visualization gives the impression of exact knowledge about begin, end, and duration of tasks. This can easily lead to the situation of uncertainties that are buried in oblivion. PERT charts, on the other hand, allow for the integration of uncertainties. But this can only be done textually which makes spotting problematic areas and comparison at a glance very hard. Furthermore, mostly only "slack times" (time between tasks) is considered, not variations of begin, end, or duration.

A way of improving this situation is to make knowledge about uncertainties visible and thus explicit. "People are visual creatures. Most people, 
if not all, perform better when things are pictorially associated. ... Instead of using their cognitive powers to figure things out, managers can perceive visually what they need to perceive about the nature of all the data involved." [14] This makes it easier to deal with temporal uncertainties and keeps them in the mind of project managers.

In Section 2 we introduce a novel glyph called PlanningLine that makes temporal uncertainties visible. PlanningLines allow the representation of a complex set of time attributes treating begin, end, and duration as intervals rather than instants. PlanningLines were originally designed to ease medical treatment planning [1] and have been modified to meet the demands of project management. In Section 3 we empirically evaluate PlanningLines with the well-known PERT technique to investigate the impact of the novel representation.

\section{PlanningLine Glyph}

Before presenting our visual representation in detail, we introduce the design goals that drove the development. In principle, these design goals can be divided into two major areas - single-glyph-related and multiple-glyph-related goals. Particularly, goals related to a single glyph are to provide a visual representation of temporal indeterminacies of a single activity, facilitate the identification of (un)defined attributes, support in maintaining logical constraints, and to give a visual impression of how distinctive the individual and overall uncertainties are. Goals related to multiple glyphs (parts of a project plan or complete plans) are foremost to support the identification of critical areas, facilitate the understanding of activity interrelationships and hierarchy as well as the comparison of activities. Overall design goals are to provide an intuitive visual representation with low learning effort that can easily be integrated into current techniques.

\subsection{Design Concept}

For our glyph, the concept of LifeLines [10] has been extended to enable the display of hierarchical decomposition as well as a set of complex time annotations to reflect temporal indeterminacies. These new visual elements are called PlanningLines and allow for the interactive representation of temporal intervals with hierarchical decomposition and simple element characteristics. The glyph consists of two encapsulated bars, representing minimum and maximum duration, that are bounded by two caps that represent start and end intervals (see Figure 1).

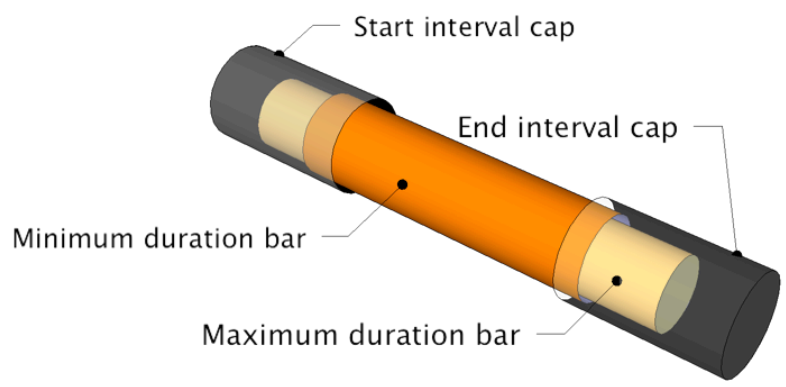

Figure 1: Glyph Concept

Temporal Attributes. For reflecting temporal uncertainties, begin, end, and duration of activities are modeled as intervals including a set of six attributes:

- start interval

o earliest starting time [EST]

o latest starting time [LST]

- end interval

o earliest finishing time [EFT]

$\circ$ latest finishing time [LFT]

- duration

o minimum duration [minDu]

o maximum duration [maxDu]

This implies that the actual start of an activity might be any instant within the start interval and an activity's end any instant within the end interval while the duration of the activity might be any span between minimum and maximum duration. Moreover, the start and end attributes can either be defined absolutely on the time scale (e.g., Dec $10^{\text {th }}$ ) or as shifts relative to a reference point (e.g., two days after the end of Activity A).

Visual Representation. The glyph itself consists of three main parts: The start cap on the left, the end cap on the right, and the duration bars in between (see Figure 2). The caps are drawn in black to emphasize their fixed position. The bars in contrary are colored whereas the color of the maximum duration bar has equal hue and saturation but higher brightness as the minimum duration bar.

Mental Model. In order to facilitate a straight forward explanation of the visual representation we use a simple mental model. The two black caps representing begin and end interval are mounted at the time scale. These caps are holding two encapsulated bars that represent minimum and maximum duration (see Figure 1). Furthermore, the bars can be shifted within the constraints of the two mounted caps. 


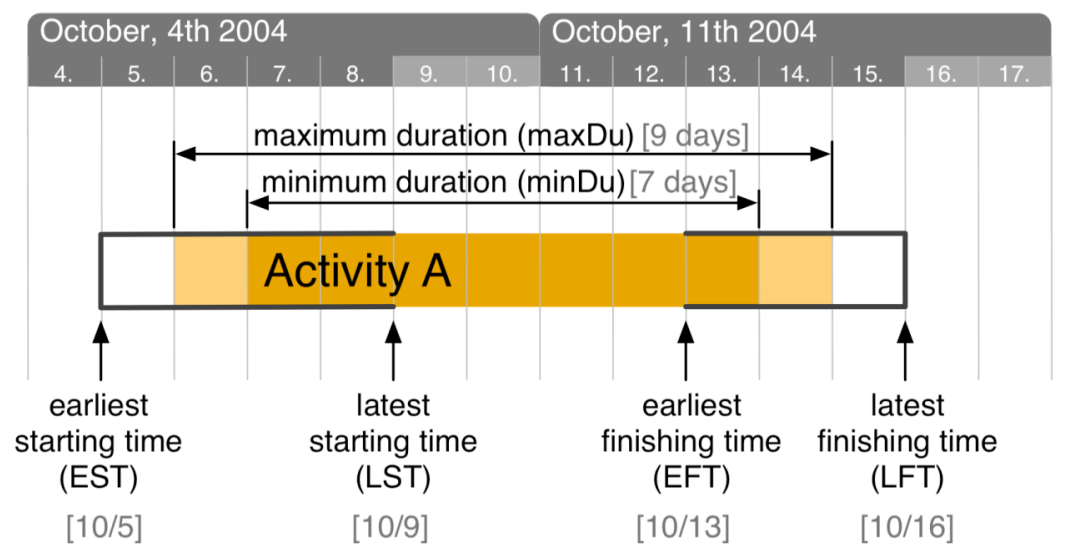

Figure 2: Represented Time Attributes

\subsection{Attribute Constraints}

For maintaining a valid attribute set, a number of logical constraints have to be followed:

1. The interval between the latest starting time [LST] and the earliest finishing time [EFT] defines the smallest possible and the interval between the earliest starting time [EST] and the latest finishing time [LFT] defines the largest possible time window for the duration of an activity.

2. For each single time point in the starting interval [EST, LST], there must exist at least one duration out of [minDu, maxDu], which allows the finishing interval [EFS, LFS] to be reached.

3. Each single time point in the finishing interval [EFT, LFT] must be reachable by at least one duration out of [minDu, maxDu] from the starting interval [EST, LST].

4. Each duration must connect one instant in the starting interval with one instant in the finishing interval.

5. $\mathrm{EST} \leq \mathrm{LST}, \mathrm{EFT} \leq \mathrm{LFT}, \mathrm{EST} \leq \mathrm{EFT}, \mathrm{LST} \leq$ LFT, $\operatorname{minDu} \leq \operatorname{maxDu}$

Our glyph helps to maintain these constraints visually. First of all, the possible durations have to be longer than the interval between latest start and earliest finish [LST, EFT] - if this would not be the case, the inner bars would fall out of the holding caps. Secondly, the possible durations cannot be longer than the interval between earliest start and latest finish [EST, LFT] - otherwise, the inner bars would not fit into the caps. Furthermore, the inner bars have to be long enough to reach the end cap if shifted completely to the left which satisfies constraint number two - otherwise, the bars would fall out of the right cap. Analogous, this is applied for shifting the bars to the right which satisfies constraint number three. Several other implicit constraints, as for example that the earliest finishing time, might not be before the earliest starting time can also easily be maintained and spotted visually at a glance.

\subsection{Special Constellations}

All temporal attributes can be specified optionally since they may not all be known. However, the remaining ones still have to maintain the constraints that are applicable and attributes might be calculated (e.g., the minimum duration by the interval between latest start and earliest end). Undefined attributes are not drawn at all and attributes that have been calculated are represented in lighter colors (e.g., gray instead of black for start and end interval attributes). If only the latest start (without earliest start) or earliest end (without latest end) are known, they are represented as diamonds (filled, rotated squares) that support the duration bars.

\subsection{Relative Time Attributes / Relations}

Relative attribute definitions (shifts) and relations between attributes are represented by connecting arrows similar to Gantt charts (see Figure 3). In principle we allow for the definition of end/begin (Activity 2 starts after the end of Activity 1) and start/start (Activity 2 starts after beginning of Activity 1) relationships. 


\subsection{Project Plan Example}

Figure 3 shows an example of a simple project plan of construction works using PlanningLines. When comparing the two activities "Windows / Doors" and "Roof" one can see at a glance that the activity "Windows / Doors" is afflicted with more uncertainties regarding begin and end than activity "Roof". On the one hand, "Windows / Doors" has a lot of slack time and large beginning and ending intervals whereas activity "Roof" has a fixed beginning and much less uncertainty in its finishing time. On the other hand, activity "Roof" is more indeterminate in terms of its duration compared to "Windows / Doors". The PlanningLines representation allows for a quick recognition and comparison of such constellations that would be hard to see when for example using PERT charts or completely impossible to detect with Gantt charts.

\section{Empirical Evaluation}

The challenge and need for evaluating information visualization has lately been emphasized by $\mathrm{C}$. Plaisant [11]. Accordingly, we conducted a controlled experiment comparing design elements to evaluate the usefulness of our approach. Goal of the study was to compare the performance of individuals using PlanningLine or PERT representations depicting temporal attributes and relationships of project tasks. The reason for the decision to compare PlanningLines with PERT is based on the capability of PERT to represent temporal uncertainties. The experiment design is paper-based and analogous to related studies evaluating LifeLines [7] and Paint Strips [4]. For brevity you find both experiment hypotheses and results of statistical tests in Section 3.1.8.

\subsubsection{Subjects}

The subjects in the study were 48 undergraduate and graduate students of informatics and business informatics in a usability engineering workshop. The subjects exhibit rather heterogeneous knowledge and experience levels, and had no knowledge on the PlanningLine method. Since the subjects in our study have varying degrees of experience with the PERT method, we conducted a tutorial that briefly repeated how to use PERT and introduced the new PlanningLine method to ensure a minimal common level of knowledge for the experiment, namely chart reading and basic calculation skills.

\subsubsection{Experiment Objects and Procedures}

In the following, we give a short overview of the experiment objects used in the empirical study (refer to [2] for the detailed material). All experiment participants received the following material:

1. Background Questionnaire: At the beginning of the study, a one-page questionnaire acquired the experience with PERT and other representations used in project management.

2. Answering Sheets for task solutions in three parts.

Four different versions of these sheets were available, for the combinations of the two

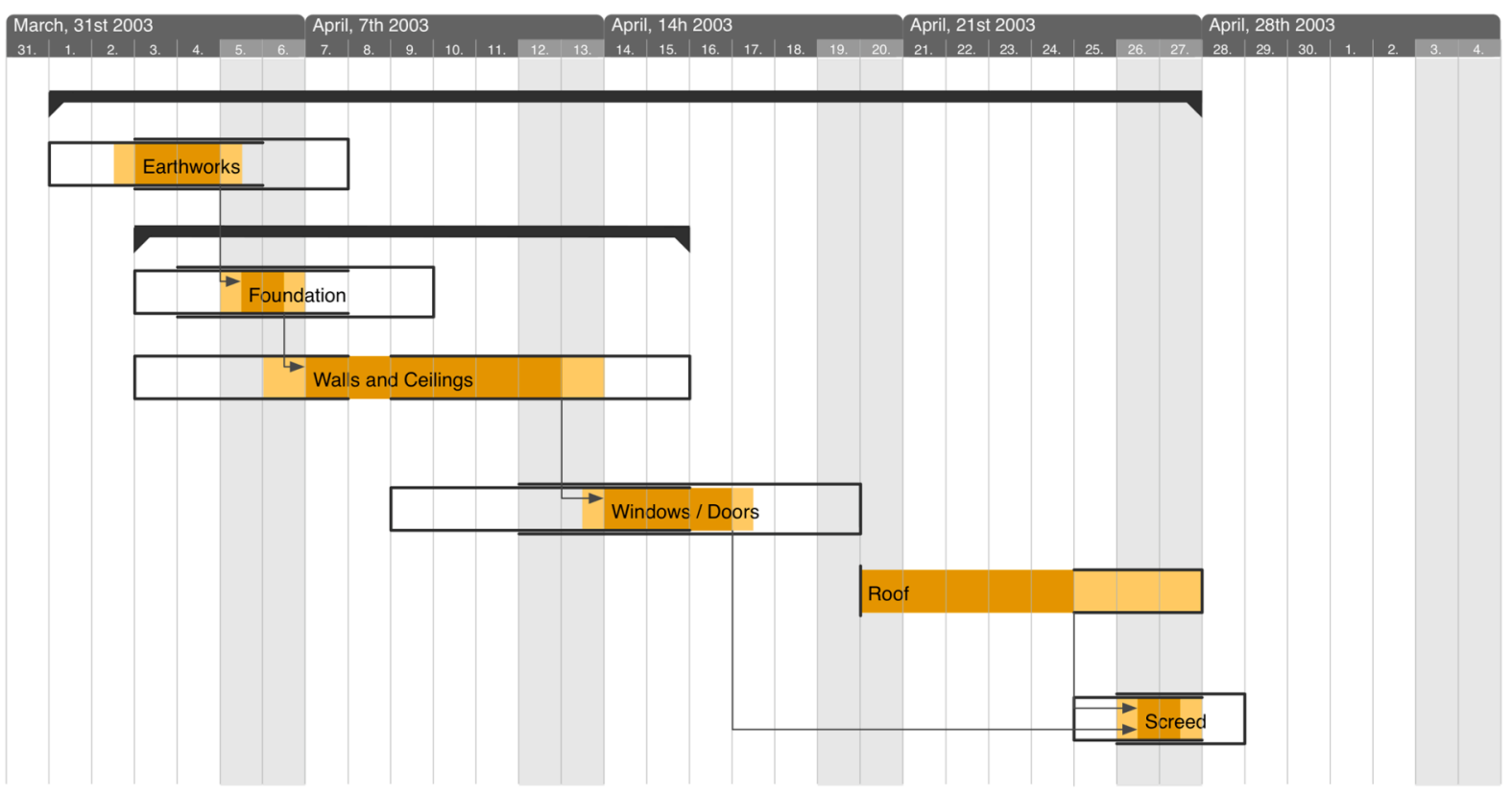

Figure 3: Example of a Simple Project Plan 
treatments (PlanningLine, PERT) and data sets $(1,2)$.

Part A: This part contained a three-page answering sheet for questions and tasks, concerning the usage of PlanningLines or PERT.

Part B: Contained an example project plan and a five-page answering sheet for questions on the project plan.

Part $C$ : A one-page answering sheet for drawing a PlanningLine/PERT chart, based on textual task description.

3. Feedback Questionnaire on the ease of use and perceived usefulness of both approaches [6].

After the tutorial that briefly repeated how to use PERT and introduced PlanningLines, the participants received the experiment material. The participants had 45 minutes to fill in the questionnaires and answering sheets. Subjects were asked to take time stamps at the start and end of each part of the answering sheet. These time stamps allow to measure the time needed to work on the tasks in a part.

\subsubsection{Experiment Design}

We randomly selected students for the two groups in the study - initial PlanningLine and PERT users. By randomization we forced unknown source of discrepancy to contribute homogeneously to the treatments, following the suggestion presented in [3]. During the experiment each individual independently worked on the experimental material. In addition to the two treatments, PlanningLine and PERT, we used two project data sets to investigate whether the treatments performed similarly with different data sets.

\subsubsection{Threats to Validity}

In every empirical study there are possible threats to the validity of the study which need to be acknowledged and mitigated with appropriate countermeasures. With the experiment design we prevented threats to internal validity: history, maturation, selection, and process conformance [13]. Regarding external validity we took a control method (PERT) that is widely used in practice and we investigated mainly cognitive abilities of subjects rather than their project management abilities. Thus using students for the study is not a problem.

\subsubsection{Experiment Variables}

The goal of the empirical study is to infer causality or to analyze relationships between variables. The dependent variables measure the effect of manipulating the independent variables [8] subject performance on number of mistakes when answering a standard questionnaire and duration for answering these questions. Independent variables are defined as factors believed to influence the results of the experiment [8], in our case the treatments (PlanningLine and PERT) and the project data sets.

\subsubsection{Data Analysis Approach}

In this paper we use the following notation to describe a combination of treatment (PlanningLine, PERT) and data sets $(1,2)$ : PlanningLine1 means using PlanningLines with data set 1 .

We used statistical differences to determine significant differences in the dependent variables' means caused by the independent variables. In most cases the parametric t-test or its non-parametric counterpart, the Mann-Whitney test can be used to compare two sample means [13]. The statistical tests were performed with an $\alpha$-level of 0.05 .

\subsubsection{Test on Similarity of Data Sets}

First we tested the similarity of performance with the two project data sets. We have four groups of combinations of treatments and data sets (PlanningLine1, PlanningLine2, PERT1, and PERT2). We evaluated the performance (relative number of mistakes and duration) of groups that use the same data set but different representation techniques (PlanningLine1-PlanningLine2, PERT1PERT2). There is no significant difference in the performance of the project data sets regarding mistakes $(p=0.501$ for PERT and $p=0.431$ for PlanningLines) and duration $(\mathrm{p}=0.601$ for PERT and $\mathrm{p}=0.401$ for PlanningLines).

After establishing that the users of different data sets perform in a similar way, we can compare the performance of different representations regardless of the data set used (PlanningLine $1+2-$ PERT1+2). The hypotheses correspond to testing the user groups and data set combinations regarding data from different parts and sections of the experiment material.

\subsubsection{Hypotheses and test results}

As t-test and Mann-Whitney test consistently showed similar results, we report the $p$ values from the t-test. The hypotheses correspond to testing the above user groups and data set combinations (PlanningLine1+2 vs. PERT1+2) regarding results from the different parts and sections of the experiment material. In the following, we state the hypothesis and the results.

1. The PlanningLine representation is as simple and intuitive to use as the PERT representation.

Regarding the performance of both mistakes $(p=0.468)$ and time $(p=0.323)$ there is no significant difference between PlanningLine and PERT users.

2. The classical PERT chart is more appropriate for answering detailed questions on single attributes of a project plan than PlanningLines. 
While PERT users make significantly fewer mistakes than PlanningLine users $(p=0.016)$, the task duration of both technologies is not significantly different $(\mathrm{p}=0.087)$.

3. The PlanningLine representation is better suited to deal with temporal uncertainties regarding the duration, start, or end of activities or plans.

PlanningLine users do not make significantly fewer mistakes than PERT users $(p=0.086)$, but the task duration of PlanningLine users is significantly shorter $(\mathrm{p}=0.012)$.

4. Possible critical sections in a project plan can be spotted easier and more correctly using PlanningLines as with PERT charts.

PlanningLine users make significantly fewer mistakes than PERT users ( $\mathrm{p}=0.089)$.

5. The layout and meaning of individual parts of the PlanningLine glyph are recalled easier as of the PERT representation.

PlanningLine users make significantly more mistakes $(p=0.000)$ and take longer than PERT users $(\mathrm{p}=0.000)$.

6. PlanningLines are perceived subjectively positive.

PlanningLines users are more content using their method than PERT users $(\mathrm{p}=0.005)$.

\subsubsection{Discussion}

Overall, the experiment results confirm our assumptions regarding PlanningLines. The fundamental assumption that PlanningLines are generally not harder to use for typical project management tasks as PERT charts was supported by the study results. Furthermore, PlanningLine users are faster in answering questions on temporal uncertainties which clearly reflects our intentions. Only the fifth hypothesis on the recall of the representation was not supported by the study results which might be caused by the fact that most subjects used PERT before. As predicted, PlanningLines are harder to use for reading exact attributes but are best for overall analysis of temporal uncertainties and can be augmented with dynamic display of explicit detail data as needed. Besides the quantifiable results, users subjectively judged PlanningLines positively.

\section{Related Work}

LifeLines [10] utilize horizontal bars to represent the temporal location and duration of data elements. They were applied for representing personal histories and patient records. For organizing the elements, socalled "facets" are introduced for grouping the data which can be expanded and collapsed. When collapsed, only a very small and geometrically as well as semantically downscaled version without textual labels is shown. Furthermore, information can be encoded via the height and color of individual bars. Additional information can be provided on demand in a linked view as for example x-ray images or the like. Due to their simplicity they are easy to understand but some important features are missing, such as the ability of depicting hierarchical data. The visualization is mainly used retrospectively for analytic and presentation tasks rather than for planning. Moreover, LifeLines cannot represent temporal indeterminacies.

With Paint Strips [4] the idea of Timelines is enriched by a painting metaphor indicating that the displayed bars are drawn by a paint roller. A paint roller at an end of a bar means that this line can expand by moving the roller until a wall is reached. This way the maximum duration and earliest start or latest end, depending on which end of the painting strip the paint rollers are attached to, are defined and indeterminacies shown. Another addition is the possibility to combine strips. The relationship of Paint Strips can be fixed, which means that if one strip moves, the other one moves in the same extent as well. This relationship is indicated graphically by connecting the involved paint rollers and attaching them to a weight at the end of a "rope" which is able to move the rollers. Paint Strips were especially developed for medical applications but can be used elsewhere as well. Due to the simplicity of the paint strip metaphor, some time annotation attributes such as durations independent of the differences between start and end points, different granularities, undefined values, or a reference point cannot be visualized.

Temporal Objects [5] were developed for depicting temporal data with different granularities. Temporal data that is defined in a coarser granularity level than the visual representation is depicted by two encapsulated bars for minimum and maximum duration with a cap at each end for the start and end intervals. Although being visually similar to PlanningLines, this technique has been developed to serve a fundamentally different purpose (granularity vs. indeterminacy), is of static nature, and less flexible.

The Time Annotation Glyph [9] is based on the same attribute set as the PlanningLine, but uses the metaphor of bars that lie on "pillars". Four vertical lines on the base specify earliest and latest starting and ending times. These pillars support a bar that represents the maximum task duration. On top of the maximum-duration bar, a minimum-duration bar lies upon two diamonds for latest start and earliest end. Furthermore, undefined parts are displayed in gray and different temporal granularities are indicated by using zigzag lines. Because of this metaphor, a few simple time-attribute constraints can be understood 
intuitively. For example, the minimum duration cannot be shorter than the interval between latest start and earliest end - if it was, the minimum duration bar would fall down between its supports. All attributes may be defined relative to a reference point that is also represented graphically. Disadvantages of Time Annotation Glyphs are their relatively steep learning curve, difficult integration into currently used and well-known techniques, and less visual cues in order to help maintaining timeattribute constraints as with PlanningLines.

\section{Conclusions}

Temporal uncertainty is a very important issue in different applications domains. To represent and visualize temporal uncertainty, we have designed and developed an innovative glyph, called PlanningLines, concerning the starting and ending times and the duration of actions or events. PlanningLines were originally designed for medical treatment planning. In this paper we illustrated how PlanningLines can be applied to the domain of project management.

Designing novel visualization methods is a necessary task to improve the communication of complex interdependencies. However, to assess the real usability of such visualization, an evaluation must be performed [11]. We conducted an empirical study comparing our designed PlanningLines with PERT charts, which demonstrated the usefulness of our approach on a cognitive level.

Acknowledgements Many thanks to Peter Messner, for reviewing the experiment design, to Gernot Goluch, Christoph Gesperger, Manuel Ganglberger, and Christoph Fleury, for their support in preparing the experiment material, and to the experiment participants. This project is supported by "Fonds zur Förderung der wissenschaftlichen Forschung - FWF" (Austrian Science Fund), grant P15467-N04.

\section{References}

[1] Wolfgang Aigner, Interactive Visualization of TimeOriented Treatment Plans and Patient Data, Master's thesis, Vienna University of Technology, Institute of Software Technology and Interactive Systems, Vienna, Austria, 2003.

[2] Wolfgang Aigner, Silvia Miksch, Bettina Thurnher, Stefan Biffl. PlanningLines Usability Studie - User Study zum Vergleich von PlanningLines und PERT Darstellung (in German), Vienna University of Technology, Institute of Software Technology and Interactive Systems, Vienna, Technical Report, Asgaard-TR-2005-3, 2005.

[3] George E. P. Box, William G. Hunter, J. Stuart Hunter, William Gordon Hunter. Statistics for Experimenters, John Wiley \& Sons. 1978.
[4] Luca Chittaro and Carlo Combi. Visualizing Queries on Databases of Temporal Histories: New Metaphors and their Evaluation. Data and Knowledge Engineering. 44(2): 239-264, 2003.

[5] Carlo Combi, Luisa Portoni, and Francesco Pinciroli. Visualizing Temporal Clinical Data on the WWW. In Proceedings of the Joint European Conference on Artificial Intelligence in Medicine and Medical Decision Making (AIMDM'99). Springer, 301-311, 1999.

[6] Fred D. Davis. Perceived Usefulness, Perceived Ease of Use and User Acceptance of Information Technology. MIS Quarterly, 13(3): 319-340. 1989.

[7] Diane Lindwarm Alonso, Anne Rose, Catherine Plaisant, and Kent L. Norman. Viewing Personal History Records: A Comparison of Tabular Format and Graphical Presentation using LifeLines. Behavior and Information Technology. 17(5): 249262, 1998.

[8] Christopher M. Lott and H. Dieter Rombach. Repeatable Software Engineering Experiments for Comparing Defect-Detection Techniques. Empirical Software Engineering 1(3), 241-277. 1996.

[9] Silvia Miksch and Robert Kosara. Communicating Time-Oriented, Skeletal Plans to Domain Experts Lucidly. In Database and Expert Systems Applications, Proceedings of the10th International Conference of Database and Expert Systems Applications (DEXA'99). Springer, 1041-1051, 1999.

[10] Catherine Plaisant, Richard Mushlin, Aaron Snyder, Jia Li, Dan Heller, and Ben Shneiderman. LifeLines: Using Visualization to Enhance Navigation and Analysis of Patient Records. In Proceedings of the 1998 American Medical Informatic Association Annual Fall Symposium. 76-80, 1998

[11] Catherine Plaisant. The Challenge of Information Visualization Evaluation, In Proceedings of the working conference on Advanced Visual Interfaces (AVI 2004). ACM, 109-116, 2004.

[12] Diana White and Joyce Fortune. Current Practice in Project Management - An Empirical Study. International Journal of Project Management. 20(1): 1-11, 2002.

[13] Claes Wohlin, Per Runeson, Martin Höst, Magnus C. Ohlsson, Björn Regnell, Anders Wesslén. Experimentation in Software Engineering - An Introduction. The Kluwer International Series in Software Engineering, Kluwer Academic Publishers. 2000.

[14] Ping Zhang and Dan Zhu. Information Visualization in Project Management and Scheduling. In Proceedings of the 4th Conference of the International Society for Decision Support Systems (ISDSS'97). 1-9, 1997. 